\title{
Sequence of Planned Assessment Schedule
}

National Cancer Institute

\section{Source}

National Cancer Institute. Sequence of Planned Assessment Schedule. NCI Thesaurus.

Code C117298.

A number that identifies the ordering relations in time of the schedules for planned assessments in a trial. 